\title{
The distribution of zeros of Epstein zeta functions over $\mathrm{GL}_{n}$
}

\author{
by \\ Riad MasRi (Bonn)
}

1. Introduction. 1. The study of the distribution of the zeros of Epstein zeta functions has a long and interesting history (see e.g. [BG, BH, BM, $\mathrm{DH}, \mathrm{E}, \mathrm{H}, \mathrm{St}]$ ). In this paper we study the distribution of the nontrivial zeros of ideal class zeta functions associated to elements in the symmetric space of $\mathrm{GL}_{n}$ over a number field $K$ (see Definition 1.1). These zeta functions have been studied extensively by Terras in [T1-T4]. They generalize the classical Epstein zeta function of a positive definite quadratic form. Furthermore, when $n=2$ they play an important role in the study of the spectral theory of the Laplacian through their relationship with $\mathrm{SL}_{2}$-Eisenstein series (see $[\mathrm{M}]$ ). We will use a method of Levinson and Montgomery [LM] to establish asymptotics for the number of nontrivial zeros up to height $T$, and asymptotics for the distribution of the nontrivial zeros with respect to the critical line. We then combine these results to study the mean value of the real parts of the nontrivial zeros. Our results generalize those of Steuding $[\mathrm{S}]$ for $K=\mathbb{Q}$.

2. We now define the zeta functions to be studied in this paper (see also [T4]). Let $n$ be a positive integer and $K$ be a number field of degree $N=r_{1}+2 r_{2}$ over $\mathbb{Q}$. The symmetric space $\mathcal{Q}_{K}^{n}$ of positive $n$-forms over $K$ consists of $r_{1}+r_{2}$-tuples $Q=\left(Q^{(1)}, \ldots, Q^{\left(r_{1}+r_{2}\right)}\right)$ of positive definite $n \times n$ matrices such that the first $r_{1}$ are real symmetric and the second $r_{2}$ are complex Hermitian. The determinant one subspace $\mathcal{S} \mathcal{Q}_{K}^{n}$ consists of $Q$ in $\mathcal{Q}_{K}^{n}$ such that $\operatorname{det}\left(Q^{(j)}\right)=1$ for $j=1, \ldots, r_{1}+r_{2}$. There is an identification

$$
\mathcal{Q}_{K}^{n} \leftrightarrow\left(\mathrm{GL}_{n}(\mathbb{R}) / \mathrm{O}(n)\right)^{r_{1}} \times\left(\mathrm{GL}_{n}(\mathbb{C}) / \mathrm{U}(n)\right)^{r_{2}}
$$

realizing $\mathcal{Q}_{K}^{n}$ as products of the rank $n-1$ symmetric spaces $\mathrm{GL}_{n}(\mathbb{R}) / \mathrm{O}(n)$ and $\mathrm{GL}_{n}(\mathbb{C}) / \mathrm{U}(n)$, respectively. The group $\mathrm{GL}_{n}(K)$ acts on $\mathcal{Q}_{K}^{n}$ by

$$
Q[A]=\left({\overline{A^{(1)}}}^{t} Q^{(1)} A^{(1)}, \ldots,{\overline{A^{\left(r_{1}+r_{2}\right)}}}^{t} Q^{\left(r_{1}+r_{2}\right)} A^{\left(r_{1}+r_{2}\right)}\right) \quad \text { for } A \in \mathrm{GL}_{n}(K),
$$

where $A^{(j)}$ means the $j$ th embedding of $K$ is applied to each entry of $A$.

2000 Mathematics Subject Classification: Primary 11M41.

Key words and phrases: Epstein zeta function, symmetric space, zero distribution. 
Let $\mathcal{O}_{K}$ be the ring of integers and $U_{K}$ be the group of units of $K$. Given an integral ideal $\mathfrak{a}$ in $K$, let $[\mathfrak{a}]$ be its ideal class and $N_{K / \mathbb{Q}}(\mathfrak{a})=\left|\mathcal{O}_{K}: \mathfrak{a}\right|$ be its norm. Two elements $a$ and $a^{\prime}$ in $\mathfrak{a}^{n}$ are said to be associate modulo $U_{K}$ if there exists a unit $\varepsilon$ in $U_{K}$ such that $a=\varepsilon a^{\prime}$. Let $\mathcal{R}\left(\mathfrak{a}^{n}\right)$ be a complete set of nonzero, nonassociate elements in $\mathfrak{a}^{n}$. Finally, let $S_{\infty}$ be a collection of $r_{1}+r_{2}$ infinite places $\nu$ of $K$, and let

$$
N_{\nu}= \begin{cases}1 & \text { if } \nu \text { is real, } \\ 2 & \text { if } \nu \text { is complex. }\end{cases}
$$

Definition 1.1. The ideal class zeta function associated to a positive $n$-form $Q$ in $\mathcal{Q}_{K}^{n}$ is defined by

$$
\zeta_{n}(Q,[\mathfrak{a}], s)=N_{K / \mathbb{Q}}(\mathfrak{a})^{2 s} \sum_{a \in \mathcal{R}\left(\mathfrak{a}^{n}\right)} N(Q[a])^{-s}, \quad \operatorname{Re} s>n / 2,
$$

where

$$
N(Q[a])=\prod_{\nu \in S_{\infty}} Q^{(\nu)}\left[a^{(\nu)}\right]^{N_{\nu}} .
$$

Remarks. (a) The factor $N_{K / \mathbb{Q}}(\mathfrak{a})^{2 s}$ ensures that $\zeta_{n}(Q,[\mathfrak{a}], s)$ depends only on the ideal class $[\mathfrak{a}]$.

(b) It is a consequence of the definition that $\zeta_{n}(Q,[\mathfrak{a}], s)$ is automorphic with respect to the action of $\mathrm{GL}_{n}\left(\mathcal{O}_{K}\right)$ on $\mathcal{Q}_{K}^{n}$, i.e.

$$
\zeta_{n}(Q[A],[\mathfrak{a}], s)=\zeta_{n}(Q,[\mathfrak{a}], s) \quad \text { for all } A \in \mathrm{GL}_{n}\left(\mathcal{O}_{K}\right) .
$$

(c) When $K=\mathbb{Q}$ the zeta function $\zeta_{n}(Q,[\mathfrak{a}], s)$ equals the classical Epstein zeta function of a positive-definite real symmetric matrix $Q$,

$$
\zeta_{n}(Q,[\mathbb{Z}], s)=\frac{1}{2} \sum_{a \in \mathbb{Z}^{n} \backslash\{0\}} Q[a]^{-s}, \quad \operatorname{Re} s>n / 2 .
$$

(d) The zeta functions $\zeta_{2}(Q,[\mathfrak{a}], s)$ can be used to compute the scattering matrix and scattering determinant associated to the functional equation satisfied by the $\mathrm{SL}_{2}$-Eisenstein series corresponding to the cusps of the noncompact, finite volume quotient $X=\mathrm{SL}_{2}\left(\mathcal{O}_{K}\right) \backslash \mathcal{S} \mathcal{Q}_{K}^{2}$ (see $[\mathrm{M}]$ ). One can combine this information with the Selberg trace formula for $X$ to establish Weyl's law for the function $N(T)$ which counts the number of eigenvalues $\lambda \leq T$ corresponding to cuspidal eigenfunctions for the Laplacian $\Delta$ in $L^{2}(X)$ (see [R, Sa1, Sa2]).

3. We will make repeated use of the functional equation for $\zeta_{n}(Q,[\mathfrak{a}], s)$ (for the proof see $[\mathrm{M}]$ ).

THEOREM 1.2. Let $A=2^{-r_{2}} d_{K}^{1 / 2} \pi^{-N / 2}$, where $d_{K}$ is the absolute value of the discriminant of $K$, and let $\mathfrak{a}^{*}$ be the dual of $\mathfrak{a}$. Then $\zeta_{n}(Q,[\mathfrak{a}], s)$ is 
meromorphic on $\mathbb{C}$ with a simple pole at $s=n / 2$, and

$$
\begin{aligned}
& A^{2 s} \Gamma(s)^{r_{1}} \Gamma(2 s)^{r_{2}} \zeta_{n}(Q,[\mathfrak{a}], s) \\
= & \|Q\|^{-1 / 2} A^{2(n / 2-s)} \Gamma(n / 2-s)^{r_{1}} \Gamma(2(n / 2-s))^{r_{2}} \zeta_{n}\left(Q^{-1},\left[\mathfrak{a}^{*}\right], n / 2-s\right),
\end{aligned}
$$

where

$$
\|Q\|=\prod_{\nu \in S_{\infty}}\left|\operatorname{det}\left(Q^{(\nu)}\right)\right|^{N_{\nu}}
$$

is the norm of the determinant of $Q$.

It follows from the functional equation (1.1) that $\zeta_{n}(Q,[\mathfrak{a}], s)$ has zeros at $m=-1,-2, \ldots$ We refer to these as trivial zeros and to all other zeros $\varrho=\beta+i \gamma$ as nontrivial zeros.

4. We now state our main results. Let

$$
N(Q,[\mathfrak{a}], T)=|\{\varrho=\beta+i \gamma:|\gamma| \leq T\}|
$$

count the number of nontrivial zeros $\varrho$ with $|\gamma| \leq T$. Here the zeros are counted according to multiplicities. Let

$$
\begin{aligned}
& \operatorname{m}(Q,[\mathfrak{a}])=\min \left\{N(Q[a]) / N_{K / \mathbb{Q}}(\mathfrak{a})^{2}: a \in \mathcal{R}\left(\mathfrak{a}^{n}\right)\right\} \\
& \mathrm{M}(Q,[\mathfrak{a}])=\left|\left\{a \in \mathcal{R}\left(\mathfrak{a}^{n}\right): N(Q[a]) / N_{K / \mathbb{Q}}(\mathfrak{a})^{2}=\operatorname{m}(Q,[\mathfrak{a}])\right\}\right| .
\end{aligned}
$$

It is not difficult to show that $\operatorname{m}(Q,[\mathfrak{a}])$ and $\mathrm{M}(Q,[\mathfrak{a}])$ depend only on the ideal class $[\mathfrak{a}]$.

In the following theorem we establish asymptotics for the number of nontrivial zeros up to height $T$, and asymptotics for the distribution of the nontrivial zeros with respect to the critical line.

TheOREM 1.3. As $T \rightarrow \infty$,

$$
N(Q,[\mathfrak{a}], T)=\frac{2 N T}{\pi} \log \left\{\frac{T}{\pi e \sqrt{\mathrm{m}(Q,[\mathfrak{a}]) \mathrm{m}\left(Q^{-1},\left[\mathfrak{a}^{*}\right]\right)}}\right\}+O(\log T)
$$

and

$$
\sum_{|\gamma| \leq T}\left(\beta-\frac{n}{4}\right)
$$

$=-\frac{N T}{\pi} \log \left\{\|Q\|^{1 / 2 N}\left(\frac{\mathrm{M}(Q,[\mathfrak{a}])}{\mathrm{M}\left(Q^{-1},\left[\mathfrak{a}^{*}\right]\right)}\right)^{1 / N}\left(\frac{\mathrm{m}\left(Q^{-1},\left[\mathfrak{a}^{*}\right]\right)}{\mathrm{m}(Q,[\mathfrak{a}])}\right)^{n / 4}\right\}+O(\log T)$.

Equation (1.3) measures, in some sense, the asymmetries in the distribution of the nontrivial zeros of $\zeta_{n}(Q,[\mathfrak{a}], s)$ with respect to the critical line. To see this, consider the constant

$$
\Sigma(Q,[\mathfrak{a}])=\log \left\{\|Q\|^{1 / 2 N}\left(\frac{\mathrm{M}(Q,[\mathfrak{a}])}{\mathrm{M}\left(Q^{-1},\left[\mathfrak{a}^{*}\right]\right)}\right)^{1 / N}\left(\frac{\mathrm{m}\left(Q^{-1},\left[\mathfrak{a}^{*}\right]\right)}{\mathrm{m}(Q,[\mathfrak{a}])}\right)^{n / 4}\right\} .
$$


If $K=\mathbb{Q}$ and $n=2$, so that $Q$ corresponds to a binary quadratic form, then $\zeta_{2}(Q,[\mathbb{Z}], s)$ is a constant multiple of $\zeta_{2}\left(Q^{-1},[\mathbb{Z}], s\right)$, which implies via the functional equation (1.1) that there is a bijection between the zeros $\varrho$ and $1-\varrho$. It follows immediately that $\Sigma(Q,[\mathbb{Z}])=0$. Note, however, that it is possible that there are configurations of a few asymmetrically distributed zeros such that $\Sigma(Q,[\mathfrak{a}])=0$. It is not difficult to construct examples with $\Sigma(Q,[\mathbb{Z}]) \neq 0($ see $[\mathrm{S}])$.

By combining (1.2) and (1.3) we find that the mean value of the real parts of the nontrivial zeros of $\zeta_{n}(Q,[\mathfrak{a}], s)$ exists and equals $n / 4$, and thus the possible asymmetries in the distribution of the nontrivial zeros are small when compared with the total number of zeros.

Corollary 1.4. As $T \rightarrow \infty$,

$$
\frac{1}{N(Q,[\mathfrak{a}], T)} \sum_{|\gamma| \leq T} \beta=\frac{n}{4}+O\left(\frac{1}{\log T}\right) .
$$

Acknowledgments. I would like to thank the Max-Planck-Institut für Mathematik in Bonn for support during the preparation of this work.

2. Proof of Theorem 1.3. This proof is based on a method of Levinson and Montgomery $[\mathrm{LM}]$ used to study the distribution of the zeros of the derivatives of the Riemann zeta function. See also the elaboration on this method in [S].

We will use the following lemma due to Littlewood [Li].

Lemma 2.1. Let $A<B$ and let $f(s)$ be analytic on $\mathcal{R}=\{s \in \mathbb{C}: A \leq$ $\sigma:=\operatorname{Re} s \leq B,|t| \leq T\}$. Suppose that $f(s)$ does not vanish on the right edge $\sigma=B$ of $\mathcal{R}$. Let $\mathcal{R}^{\prime}$ be $\mathcal{R}$ minus the union of the horizontal cuts from the zeros of $f$ in $\mathcal{R}$ to the left edge of $\mathcal{R}$, and choose a single-valued branch of $\log f(s)$ in the interior of $\mathcal{R}^{\prime}$. Denote by $\mathrm{v}(\sigma, T)$ the number of zeros $\varrho=\beta+i \gamma$ of $f(s)$ inside $\mathcal{R}$ with $\beta>\sigma$ including zeros with $\gamma=T$ but not those with $\gamma=-T$. Then

$$
\int_{\partial \mathcal{R}} \log f(s) d s=-2 \pi i \int_{A}^{B} \mathrm{v}(\sigma, T) d \sigma .
$$

See [T, Section 9.9] for a proof.

Define

$$
Z_{n}(Q,[\mathfrak{a}], s)=\frac{\mathrm{m}(Q,[\mathfrak{a}])^{s}}{\mathrm{M}(Q,[\mathfrak{a}])} \zeta_{n}(Q,[\mathfrak{a}], s) .
$$

Then $Z_{n}(Q,[\mathfrak{a}], s)$ has the same zeros as $\zeta_{n}(Q,[\mathfrak{a}], s)$, and for $\sigma>n / 2$,

$$
Z_{n}(Q,[\mathfrak{a}], s)=1+\frac{1}{\mathrm{M}(Q,[\mathfrak{a}])} \sum_{\substack{a \in \mathcal{R}\left(\mathfrak{a}^{n}\right) \\ N(Q[a]) / N_{K / \mathbb{Q}}(\mathfrak{a})^{2}>\mathrm{m}(Q,[\mathfrak{a}])}}\left(\frac{\mathrm{m}(Q,[\mathfrak{a}])}{N_{K / \mathbb{Q}}(\mathfrak{a})^{-2} N(Q[a])}\right)^{s} .
$$


Let

$$
\mathrm{m}_{2}(Q,[\mathfrak{a}])=\inf \left\{a \in \mathcal{R}\left(\mathfrak{a}^{n}\right): N(Q[a]) / N_{K / \mathbb{Q}}(\mathfrak{a})^{2}>\mathrm{m}(Q,[\mathfrak{a}])\right\} .
$$

Again, it is not difficult to show that $\mathrm{m}_{2}(Q,[\mathfrak{a}])$ depends only on the ideal class $[\mathfrak{a}]$. Then

$$
Z_{n}(Q,[\mathfrak{a}], s)=1+C(Q,[\mathfrak{a}]) \lambda^{-s}+o\left(\lambda^{-\sigma}\right) \quad \text { as } \sigma \rightarrow \infty,
$$

where $C(Q,[\mathfrak{a}])>0$ is a constant, and

$$
\lambda=\mathrm{m}_{2}(Q,[\mathfrak{a}]) / \mathrm{m}(Q,[\mathfrak{a}])>1 .
$$

Consequently,

$$
Z_{n}(Q,[\mathfrak{a}], s) \rightarrow 1 \quad \text { as } \sigma \rightarrow \infty,
$$

so there exists a zero-free region for $\zeta_{n}(Q,[\mathfrak{a}], s)$ for $\sigma$ sufficiently large. Similarly, there exists a zero-free region for $\zeta_{n}\left(Q^{-1},\left[\mathfrak{a}^{*}\right], s\right)$ for $\sigma$ sufficiently large. It follows from the functional equation (1.1) that there exists a constant $B>0$ such that $\zeta_{n}(Q,[\mathfrak{a}], s)$ and $\zeta_{n}\left(Q^{-1},\left[\mathfrak{a}^{*}\right], s\right)$ have no nontrivial zeros outside of the strip $-B<\sigma<B$.

Define the function

$$
f(s)=(s-n / 2) Z_{n}(Q,[\mathfrak{a}], s) .
$$

Then $f(s)$ is entire and has the same zeros as $\zeta_{n}(Q,[\mathfrak{a}], s)$. Let $b>B$ be sufficiently large. Form the rectangle $\mathcal{R}$ with vertices $-b \pm i T, B \pm i T$, and assume that $f(s)$ does not vanish on $\partial \mathcal{R}$. In what follows let $\varrho=\beta+i \gamma$ denote any zero of $\zeta_{n}(Q,[\mathfrak{a}], s)$ (for any $T$ there are at most $b$ trivial zeros of $\zeta_{n}(Q,[\mathfrak{a}], s)$ inside $\mathcal{R}$ and thus their contribution is negligible).

We now apply Lemma 2.1 to the function $f(s)$. First, observe that

$$
\int_{-b}^{B} \mathrm{v}(\sigma, T) d \sigma
$$

is the total length of the horizontal cuts from the zeros of $f(s)$ in $\mathcal{R}$ to the left edge of $\mathcal{R}$. Then

$$
\int_{-b}^{B} \mathrm{v}(\sigma, T) d \sigma=\sum_{|\gamma| \leq T}(\beta+b),
$$

so that by Lemma 2.1,

$$
i \int_{\partial \mathcal{R}} \log f(s) d s=2 \pi \sum_{|\gamma| \leq T}(\beta+b) .
$$

Next, write

$$
i \int_{\partial \mathcal{R}} \log f(s) d s=i \int_{\partial \mathcal{R}} \log (s-n / 2) d s+i \int_{\partial \mathcal{R}} \log Z_{n}(Q,[\mathfrak{a}], s) d s .
$$


By Lemma 2.1,

$$
i \int_{\partial \mathcal{R}} \log (s-n / 2) d s=2 \pi(n / 2+b) .
$$

Furthermore (see also [T, Section 9.9, equation (9.9.2)]),

$$
\begin{aligned}
i \int_{\partial \mathcal{R}} \log & Z_{n}(Q,[\mathfrak{a}], s) d s \\
= & \int_{-T}^{T} \log Z_{n}(Q,[\mathfrak{a}],-b+i t) d t-\int_{-T}^{T} \log Z_{n}(Q,[\mathfrak{a}], B+i t) d t \\
& \quad+i \int_{-b}^{B}\left\{\log Z_{n}(Q,[\mathfrak{a}], \sigma-i T)-\log Z_{n}(Q,[\mathfrak{a}], \sigma+i T)\right\} d \sigma .
\end{aligned}
$$

By (2.3)-(2.5), the right hand side of (2.6) is real. Expand the right hand side of (2.6) and let the imaginary part equal zero to express (2.3) in the form

$$
\begin{aligned}
& 2 \pi \sum_{|\gamma| \leq T}(\beta+b) \\
= & \int_{-T}^{T} \log \left|Z_{n}(Q,[\mathfrak{a}],-b+i t)\right| d t-\int_{-T}^{T} \log \left|Z_{n}(Q,[\mathfrak{a}], B+i t)\right| d t \\
& -\int_{-b}^{B} \arg Z_{n}(Q,[\mathfrak{a}], \sigma-i T) d \sigma+\int_{-b}^{B} \arg Z_{n}(Q,[\mathfrak{a}], \sigma+i T) d \sigma+O(1) .
\end{aligned}
$$

From (2.2),

$$
\log Z_{n}(Q,[\mathfrak{a}], s) \sim C(Q,[\mathfrak{a}]) \lambda^{-s} \quad \text { as } \sigma \rightarrow \infty,
$$

thus for sufficiently large $B$,

$$
\int_{-T}^{T} \log \left|Z_{n}(Q,[\mathfrak{a}], B+i t)\right| d t=O(1)
$$

as $T \rightarrow \infty$.

We now estimate the integral

$$
\int_{-T}^{T} \log \left|Z_{n}(Q,[\mathfrak{a}],-b+i t)\right| d t .
$$

From (2.1),

$$
\begin{aligned}
& \log \left|Z_{n}(Q,[\mathfrak{a}],-b+i t)\right| \\
& \quad=-b \log \mathrm{m}(Q,[\mathfrak{a}])-\log \mathrm{M}(Q,[\mathfrak{a}])+\log \left|\zeta_{n}(Q,[\mathfrak{a}],-b+i t)\right| .
\end{aligned}
$$


Furthermore, from the functional equation (1.1),

$$
\begin{aligned}
\log \left|\zeta_{n}(Q,[\mathfrak{a}],-b+i t)\right|= & -\frac{1}{2} \log \|Q\|+(n+4 b) \log A \\
& +r_{1}\{\log |\Gamma(n / 2+b-i t)|-\log |\Gamma(-b+i t)|\} \\
& +r_{2}\{\log |\Gamma(n+2 b-2 i t)|-\log |\Gamma(-2 b+2 i t)|\} \\
& +\log \left|\zeta_{n}\left(Q^{-1},\left[\mathfrak{a}^{*}\right], n / 2+b-i t\right)\right| .
\end{aligned}
$$

By Stirling's formula,

$$
\log |\Gamma(\sigma+i t)|=\left(\sigma-\frac{1}{2}\right) \log |t|-\frac{\pi}{2}|t|+\frac{1}{2} \log (2 \pi)+O\left(|t|^{-1}\right)
$$

for $|t| \geq 1$ and $\sigma$ bounded. Using (2.9) we obtain, after a calculation,

$$
\begin{aligned}
\int_{-T}^{T} \log \left|Z_{n}(Q,[\mathfrak{a}],-b+i t)\right| d t \\
=r_{1}(n+4 b) \int_{1}^{T} \log t d t+r_{2}(n+4 b) \int_{1}^{2 T} \log t d t \\
\quad-T\{\log \|Q\|-2(n+4 b) \log A+2 b \log \mathrm{m}(Q,[\mathfrak{a}])+2 \log \mathrm{M}(Q,[\mathfrak{a}])\} \\
\quad+\int_{-T}^{T} \log \left|\zeta_{n}\left(Q^{-1},\left[\mathfrak{a}^{*}\right], n / 2+b-i t\right)\right| d t+O(\log T) .
\end{aligned}
$$

From (2.1),

$$
\begin{aligned}
& \int_{-T}^{T} \log \left|\zeta_{n}\left(Q^{-1},\left[\mathfrak{a}^{*}\right], n / 2+b-i t\right)\right| d t \\
& =\int_{-T}^{T} \log \left|Z_{n}\left(Q^{-1},\left[\mathfrak{a}^{*}\right], n / 2+b-i t\right)\right| d t \\
& \quad+T\left\{2 \log \mathrm{M}\left(Q^{-1},\left[\mathfrak{a}^{*}\right]\right)-(n+2 b) \log \mathrm{m}\left(Q^{-1},\left[\mathfrak{a}^{*}\right]\right)\right\} .
\end{aligned}
$$

The integral on the right hand side of (2.10) can be estimated as in (2.8). Furthermore, it follows from

$$
\int_{1}^{T} \log t d t=T \log T+T \log \left(e^{-1}\right)+O(1)
$$

and

$$
\log A=\frac{r_{1}}{2} \log \left(\pi^{-1}\right)+r_{2} \log \left(2^{-1} \pi^{-1}\right)+O(1)
$$

that 


$$
\begin{array}{rl}
r_{1}(n+4 b) \int_{1}^{T} \log t d t+r_{2}(n+4 b) \int_{1}^{2 T} \log t & d t+2 T(n+4 b) \log A \\
& =N(n+4 b) T \log \frac{T}{\pi e}+O(1)
\end{array}
$$

Combining the preceding analysis yields

$$
\begin{aligned}
& \int_{-T}^{T} \log \left|Z_{n}(Q,[\mathfrak{a}],-b+i t)\right| d t \\
= & N(n+4 b) T \log \frac{T}{\pi e}-T\left\{2 \log \mathrm{M}(Q,[\mathfrak{a}])-2 \log \mathrm{M}\left(Q^{-1},\left[\mathfrak{a}^{*}\right]\right)+\log \|Q\|\right. \\
+ & \left.2 b \log \mathrm{m}(Q,[\mathfrak{a}])+(n+2 b) \log \mathrm{m}\left(Q^{-1},\left[\mathfrak{a}^{*}\right]\right)\right\}+O(\log T)
\end{aligned}
$$

as $T \rightarrow \infty$.

We now estimate the integrals

$$
\int_{-b}^{B} \arg Z_{n}(Q,[\mathfrak{a}], \sigma \pm i T) d \sigma .
$$

As remarked in $[\mathrm{LM}]$, integrals of this type can be estimated by bounding the number of zeros of $\operatorname{Re} \zeta_{n}(Q,[\mathfrak{a}], \sigma+i T)$ using Jensen's formula. Arguing as in $[\mathrm{S}]$, we first reduce the problem to bounding the number of zeros. Suppose that $\operatorname{Re} \zeta_{n}(Q,[\mathfrak{a}], \sigma+i T)$ has $M$ zeros for $-b \leq \sigma \leq B$. Divide $[-b, B]$ into at most $M+1$ subintervals in each of which $\operatorname{Re} Z_{n}(Q,[\mathfrak{a}], \sigma+i T)$ is of constant sign. The variation of $\arg Z_{n}(Q,[\mathfrak{a}], \sigma+i T)$ is at most $\pi$ in each subinterval, thus

$$
\left|\arg Z_{n}(Q,[\mathfrak{a}], \sigma+i T)\right| \leq(M+1) \pi .
$$

We now estimate $M$ by an application of Jensen's formula. Let

$$
g(z)=\frac{1}{2}\left\{Z_{n}(Q,[\mathfrak{a}], z+i T)+\overline{Z_{n}(Q,[\mathfrak{a}], \bar{z}+i T)}\right\} .
$$

Then $g(\sigma)=\operatorname{Re} Z_{n}(Q,[\mathfrak{a}], \sigma+i T)$. Let $R=b+B$ and choose $T$ so that $T>$ $2 R$. Because $\operatorname{Im}(z+i T)>0$ in the open disc $|z-B|<T, Z_{n}(Q,[\mathfrak{a}], z+i T)$, and hence $g(z)$, is analytic in $|z-B|<T$ (that is, we avoid the pole at $n / 2$ ).

Let $n(r)$ denote the number of zeros of $g(z)$ in the closed disc $|z-B| \leq r$. A simple estimation yields

$$
\int_{0}^{2 R} \frac{n(r)}{r} d r \geq n(R) \int_{R}^{2 R} \frac{d r}{r}=n(R) \log 2
$$

and by Jensen's formula,

$$
\int_{0}^{2 R} \frac{n(r)}{r} d r=\frac{1}{2 \pi} \int_{0}^{2 \pi} \log \left|g\left(B+2 R e^{i \theta}\right)\right| d \theta-\log |g(B)|
$$


so that

$$
n(R) \leq \frac{1}{2 \pi \log 2} \int_{0}^{2 \pi} \log \left|g\left(B+2 R e^{i \theta}\right)\right| d \theta-\frac{\log |g(B)|}{\log 2} .
$$

It follows from (2.2) that $\log |g(B)|$ is bounded. Furthermore, from the Phragmén-Lindelöf theorem we obtain, in any strip of bounded width,

$$
\zeta_{n}(Q,[\mathfrak{a}], s)=O\left(|t|^{c(Q,[\mathfrak{a}], \sigma)}\right), \quad|t| \rightarrow \infty,
$$

for a constant $c(Q,[\mathfrak{a}], \sigma)>0$. The same estimate holds for $g(z)$. Therefore,

$$
\int_{0}^{2 \pi} \log \left|g\left(B+2 R e^{i \theta}\right)\right| d \theta=O(\log T)
$$

which implies that $n(R)=O(\log T)$. Because $(-b, B)$ is contained in the disc $|z-B| \leq R$, we obtain $M \leq n(R)$. A similar argument can be made for $\arg Z_{n}(Q,[\mathfrak{a}], \sigma-i T)$. Finally, we obtain from (2.12) the estimate

$$
\int_{-b}^{B}\left|\arg Z_{n}(Q,[\mathfrak{a}], \sigma \pm i T)\right| d \sigma=O(\log T) .
$$

Combining (2.8), (2.11), and (2.13), we get in (2.7), after a tedious but straightforward algebraic manipulation,

$$
\begin{aligned}
\sum_{|\gamma| \leq T}(\beta+b)= & \left(b+\frac{n}{4}\right) \frac{2 N T}{\pi} \log \left\{\frac{T}{\pi e \sqrt{\mathrm{m}(Q,[\mathfrak{a}]) \mathrm{m}\left(Q^{-1},\left[\mathfrak{a}^{*}\right]\right)}}\right\} \\
& -\frac{N T}{\pi} \log \left\{\|Q\|^{1 / 2 N}\left(\frac{\mathrm{M}(Q,[\mathfrak{a}])}{\mathrm{M}\left(Q^{-1},\left[\mathfrak{a}^{*}\right]\right)}\right)^{1 / N}\left(\frac{\mathrm{m}\left(Q^{-1},\left[\mathfrak{a}^{*}\right]\right)}{\mathrm{m}(Q,[\mathfrak{a}])}\right)^{n / 4}\right\} \\
& +O(\log T)
\end{aligned}
$$

as $T \rightarrow \infty$. The assumption that $f(s)$ does not vanish on $\partial \mathcal{R}$ can be removed with an error $O(1)$. Using the identity

$$
\sum_{|\gamma| \leq T}(\beta+b)=(b+n / 4) \sum_{|\gamma| \leq T} 1+\sum_{|\gamma| \leq T}(\beta-n / 4),
$$

we obtain (1.2) and (1.3). This completes the proof.

\section{References}

[BG] P. T. Bateman and E. Grosswald, On Epstein's zeta function, Acta Arith. 9 (1964), 365-373.

[BH] E. Bombieri and D. Hejhal, On the zeros of Epstein zeta-functions, C. R. Acad. Sci. Paris Sér. I Math. 304 (1987), 213-217.

$[\mathrm{BM}]$ E. Bombieri and J. Mueller, On the zeros of certain Epstein zeta functions, Oberwolfach Reports 46 (2004), 2430-2431. 
[DH] H. Davenport and H. Heilbronn, On the zeros of certain Dirichlet series, I, II, J. London Math. Soc. 11 (1936), 181-185, 307-313.

[E] P. Epstein, Zur Theorie allgemeiner Zetafunktionen, Math. Ann. 56 (1903), 615644.

[H] D. Hejhal, Zeros of Epstein zeta functions and supercomputers, in: Proc. Internat. Congress of Mathematicians, Vol. 1, 2 (Berkeley, CA, 1986), Amer. Math. Soc., Providence, RI, 1987, 1362-1384.

[LM] N. Levinson and H. Montgomery, Zeros of the derivative of the Riemann zeta function, Acta Math. 133 (1974), 49-65.

[Li] J. E. Littlewood, On the zeros of the Riemann zeta function, Proc. Cambridge Philos. Soc. 22 (1924), 295-318.

$[\mathrm{M}] \quad$ R. Masri, On the scattering determinant for the Hilbert modular group, submitted.

[R] A. Reznikov, Eisenstein matrix and existence of cusp forms in rank one symmetric spaces, Geom. Funct. Anal. 3 (1993), 79-105.

[Sa1] P. Sarnak, On cusp forms, in: The Selberg Trace Formula and Related Topics (Brunswick, ME, 1984), Contemp. Math. 53, Amer. Math. Soc., Providence, RI, 1986, 393-407.

[Sa2] - On cusp forms. II, in: Festschrift in Honor of I. I. Piatetski-Shapiro on the Occasion of His Sixtieth Birthday, Part II (Ramat Aviv, 1989), Israel Math. Conf. Proc. 3, Weizmann, Jerusalem, 1990, 237-250.

[St] H. Stark, On the zeros of Epstein's zeta function, Mathematika 14 (1967), 47-55.

[S] J. Steuding, On the zero-distribution of Epstein zeta-functions, Math. Ann. 333 (2005), 689-697.

[T1] A. Terras, The Fourier expansion of Epstein's zeta function for totally real algebraic number fields and some consequences for Dedekind's zeta function, Acta Arith. 30 (1976), 187-197.

[T2] - The Fourier expansion of Epstein's zeta function over an algebraic number field and its consequences for algebraic number theory, ibid. 32 (1977), 37-53.

[T3] - The minima of quadratic forms and the behavior of Epstein and Dedekind zeta functions, J. Number Theory 12 (1980), 258-272.

[T4] - Harmonic Analysis on Symmetric Spaces and Applications, II, Springer, Berlin, 1988.

[T] E. Titchmarsh, The Theory of the Riemann Zeta-Function, 2nd ed., edited and with a preface by D. R. Heath-Brown, Clarendon Press, Oxford Univ. Press, New York, 1986.

Max-Planck-Institut für Mathematik

Vivatsgasse 7

53111 Bonn, Germany

E-mail: masrirm@mpim-bonn.mpg.de

Received on 5.10.2006

and in revised form on 4.2.2007 